\title{
A leishmaniose Visceral Americana no Estado de São Paulo: Situação Epidemiológica em 2001-2002
}

\author{
Vera Lucia Fonseca de Camargo-Neves ${ }^{1}$, Roberta Spínola ${ }^{2}$, Lisete Lage ${ }^{2}$
}

A Leishmaniose Visceral Americana (LVA) no Estado de São Paulo (ESP) vem ocorrendo em municípios situados na região oeste paulista, nos quais a transmissão tem feição exclusivamente urbana. A espécie envolvida com a transmissão é a Lutzomyia longipalpis tendo sido registrada pela primeira vez em área urbana, em 1997, no município de Araçatuba - SP. A comprovação da enzootia envolvendo o cão com Leishmania (Leishmania) chagasi ocorreu em 1998, seguida da humana em 1999. (Camargo-Neves e Katz 1999, Costa et al.1997, Tolezano et al. 1999). A partir de então, verifica-se a expansão da doença para outros municípios da região Oeste do ESP. Neste trabalho apresenta-se a situação epidemiológica na LVA no ESP no período de 2001 - 2002.

Para a análise utilizou-se como fonte de dados: (i) dos casos humanos, o sistema nacional de notificação de agravos - SINAN; (ii) do vetor, o sistema LV_GERAL-SUCEN, onde são compilados os dados da vigilância entomológica do Estado de São Paulo e (iii) do reservatório, as informações obtidas dos municípios e compiladas no boletim "resumo de inquérito canino", criado pela SUCEN.

Consideraram-se também para a análise, os casos notificados em 2001 e em 2002. As variáveis selecionadas foram idade, critério diagnóstico e evolução do caso, a presença de $L$. longipalpis no município e a transmissão de Leishmaniose Visceral Canina (LVC).

Como resultado verificou-se que a $L$. longipalpis, principal vetor da LVA no Estado de São Paulo, já foi detectada em 41 municípios, abrangendo as regiões administrativas de Araçatuba, Bauru, Marília e Presidente Prudente (Figura 1). A expansão do vetor vem ocorrendo lentamente, tendo sido capturado inicialmente em municípios contíguos à Araçatuba e depois naqueles que estabeleceram fluxo migratório de pessoas e mercadorias com os municípios da região de Araçatuba. 0 ambiente peridomiciliar, destacando-se o local de permanência de animais domésticos, vem sendo considerado como o mais importante de criação e de abrigo de L. longipalpis, em área urbanizada (Sherlock 1994). As fêmeas desta espécie têm demonstrado antropofilia e cinofilia (CamargoNeves et al. 2002) exercendo assim o papel de vetor da LVA.

A transmissão canina foi detectada em 29 municípios, em 2002, geralmente precedendo a detecção de casos humanos (Figura 2). Além disso, como já demonstrado anteriormente existe forte associação entre as altas taxas de prevalências caninas e a incidência de casos humanos (Camargo-Neves et al. 2001).

\begin{tabular}{|c|c|c|c|c|c|c|}
\hline \multirow{2}{*}{$\begin{array}{l}\text { Ano } \\
\text { Faixa etária }\end{array}$} & \multicolumn{3}{|c|}{2001} & \multicolumn{3}{|c|}{2002} \\
\hline & caso & óbito & LET* $^{*}$ & caso & óbito & LET* \\
\hline $0-4$ & 25 & 1 & 4,0 & 58 & 2 & 3,4 \\
\hline $5-9$ & 7 & - & - & 9 & - & - \\
\hline $10-14$ & 4 & - & - & 5 & 1 & 20,0 \\
\hline $15-19$ & 2 & - & - & 2 & - & - \\
\hline $20-29$ & 6 & - & - & 5 & - & - \\
\hline $30-39$ & 7 & - & - & 5 & - & - \\
\hline $40-49$ & 3 & - & - & 10 & 1 & 10,0 \\
\hline$>50$ & 3 & 1 & 33,3 & 20 & 9 & 45,0 \\
\hline Total & 57 & 2 & 3,5 & 114 & 13 & 11,4 \\
\hline
\end{tabular}

*LET: Letalidade (\%). Fonte: SINAN

Verificou-se a expansão da doença, além do aumento do número de casos, em todos os municípios onde a doença havia sido detectada no ano anterior (Tabela 1). Em 2002, a transmissão humana foi verificada em 14 municípios: 13 situados na região de Araçatuba e um na região de Bauru. Em quatro destes municípios: Avanhadava, Bilac, Coroados e Promissão, a transmissão foi detectada pela primeira vez em 2002 e, em dois, Birigüi e Guararapes, a doença na população humana já havia sido detectada anteriormente em 1999 e em 2000 , respectivamente. Nestes dois municípios a descontinuidade das ações de limpeza urbana e do programa de eliminação de cães infectados, detectados através de inquéritos sorológicos, foram os fatores que propiciaram a reincidência da doença humana.

O maior número de casos ocorreu na faixa etária menores de 5 anos de idade, porém os maiores coeficientes de letalidade forma observados na faixa de 50 anos e mais, estes indivíduos, na maioria das vezes, eram imunosuprimidos, devido a outras morbidades pré-existentes (Figura 3). Com relação ao critério diagnóstico verificou-se um incremento de $15 \%$ de casos com confirmação laboratorial em relação ao ano anterior (Figura 4).

O programa de controle aplicado no Estado consiste na detecção e tratamento de casos humanos, busca e eliminação de cães infectados com parasotologia ou sorologia positiva, além dos animais errantes e o controle vetorial. Também é realizada a vigilância entomológica com objetivo de detectar a L. longipalipis, através de levantamento entomológico por 
Tabela 2 - Distribuição de Casos, Óbitos e Coeficiente de Letalidade de Leishmaniose Visceral Americana por Faixa Etária. Estado de São Paulo, 2001 - 2002.

\begin{tabular}{|c|c|c|c|c|c|c|c|c|c|c|c|c|}
\hline \multirow{2}{*}{$\begin{array}{l}\text { Ano } \\
\text { Município }\end{array}$} & \multicolumn{4}{|c|}{2001} & \multicolumn{4}{|c|}{2002} & \multicolumn{4}{|c|}{ Total } \\
\hline & caso & INC** $^{*}$ & óbito & LET $^{* * * *}$ & caso & $\mathrm{INC}^{*}$ & óbito & $\mathrm{LET}^{* * *}$ & caso & INC* $^{*}$ & óbito & LET $^{* * *}$ \\
\hline Andradina & 13 & 23,2 & 1 & 7,6 & 19 & 33,9 & 4 & 22,2 & 32 & 57,1 & 5 & 31,3 \\
\hline Araçatuba & 29 & 16,6 & 2 & 6,8 & 52 & 29,8 & 6 & 11,5 & 81 & 46,4 & 8 & 50,0 \\
\hline Avanhadava & 0 & 0,0 & 0 & 0,0 & 1 & 11,0 & 0 & 0,0 & 1 & 0,0 & 0 & 0,0 \\
\hline Bilac & 0 & 0,0 & 0 & 0,0 & 1 & 15,9 & 0 & 0,0 & 1 & 15,9 & 0 & 0,0 \\
\hline Birigui & 0 & 0,0 & 0 & 0,0 & 4 & 4,0 & 1 & 25 & 4 & 4,0 & 1 & 6,3 \\
\hline Castilho & 2 & 13,3 & 0 & 0,0 & 6 & 33,2 & 0 & 0,0 & 7 & 46,5 & 0 & 0,0 \\
\hline Coroados & 0 & 0,0 & 0 & 0,0 & 1 & 22,1 & 0 & 0,0 & 1 & 22,1 & 0 & 0,0 \\
\hline Guaraçai & 1 & 11,0 & 0 & 0,0 & 1 & 11,1 & 0 & 0,0 & 2 & 22,0 & 0 & 0,0 \\
\hline Guararapes & 0 & 0,0 & 0 & 0,0 & 4 & 13,6 & 1 & 25 & 4 & 13,6 & 1 & 6,3 \\
\hline Lavínia & 1 & 19,9 & 0 & 0,0 & 2 & 39,7 & 0 & 0,0 & 3 & 59,6 & 0 & 0,0 \\
\hline Mirandópolis & 3 & 11,4 & 0 & 0,0 & 7 & 26,5 & 0 & 0,0 & 10 & 37,9 & 0 & 0,0 \\
\hline Penápolis & 3 & 5,3 & 0 & 0,0 & 8 & 14,1 & 0 & 0,0 & 11 & 19,4 & 0 & 0,0 \\
\hline Valparaíso & 5 & 26,0 & 0 & 0,0 & 5 & 26,0 & 1 & 0,0 & 10 & 52,1 & 1 & 6,3 \\
\hline Região de Araçatuba & 57 & 10,3 & 3 & 100,0 & 110 & 20,0 & 13 & 100,0 & 167 & 32,7 & 16 & 100,0 \\
\hline Promissão & 0 & 0,0 & 0 & 0,0 & 4 & 12,5 & 0 & 0,0 & 4 & 12,5 & 0 & 0,0 \\
\hline Região de Bauru & 0 & 0,0 & 0 & 0,0 & 4 & 1,1 & 0 & 0,0 & 4 & 12,5 & 0 & 0,0 \\
\hline Total & 57 & 6,2 & 3 & 100,0 & 114 & 11,9 & 13 & 100,0 & 171 & 31,5 & 16 & 100,0 \\
\hline
\end{tabular}
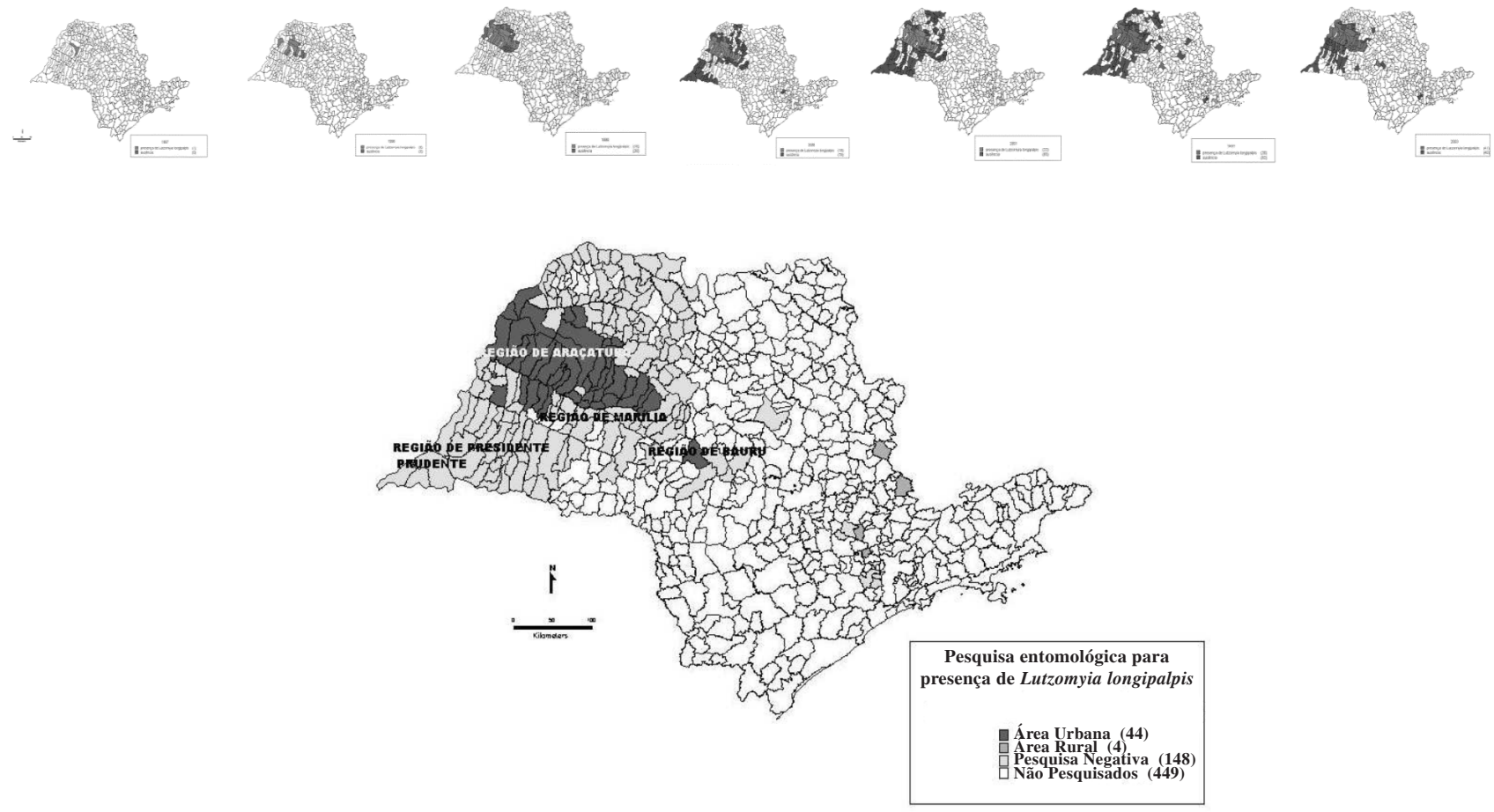

Figura 1 - Distribuição de municípios com pesquisa entomológica por ano. Estado de São Paulo, 1997 - 2003.

meio de aramdilhas elétricas, delimitando-se as áreas de sua ocorrência na região oeste do Estado de São Paulo. As ações de controle vetorial são implementadas num raio de 200 metros em torno dos casos autóctones registrados (SES 2000). Apesar dos esforços para atingir seus objetivos, a transmissão transcorre de formas subclínicas com aparecimento de casos novos registrados todos os anos. 0 município de Araçatuba é o que registra o maior número de casos entre os municípios investigados anualmente. A partir da detecção do primeiro caso de LVA no ESP, em 1999, verificou-se a expansão da doença, a medida que houve a adaptação do vetor - Lutzomyia longipalpis em novos municípios e a epizootia canina na maioria dos municípios precedeu o aparecimento dos casos humanos.

A descontinuidade das ações de controle, tanto aquelas relacionadas ao reservatório doméstico, como as relacionadas ao vetor é um fator que favorece a manutenção da transmissão. Verificou-se a necessidade de revisar as normas atualmente adotadas pelo Estado a fim de racionalizar os recursos e otimizar as ações para as áreas de maior risco 


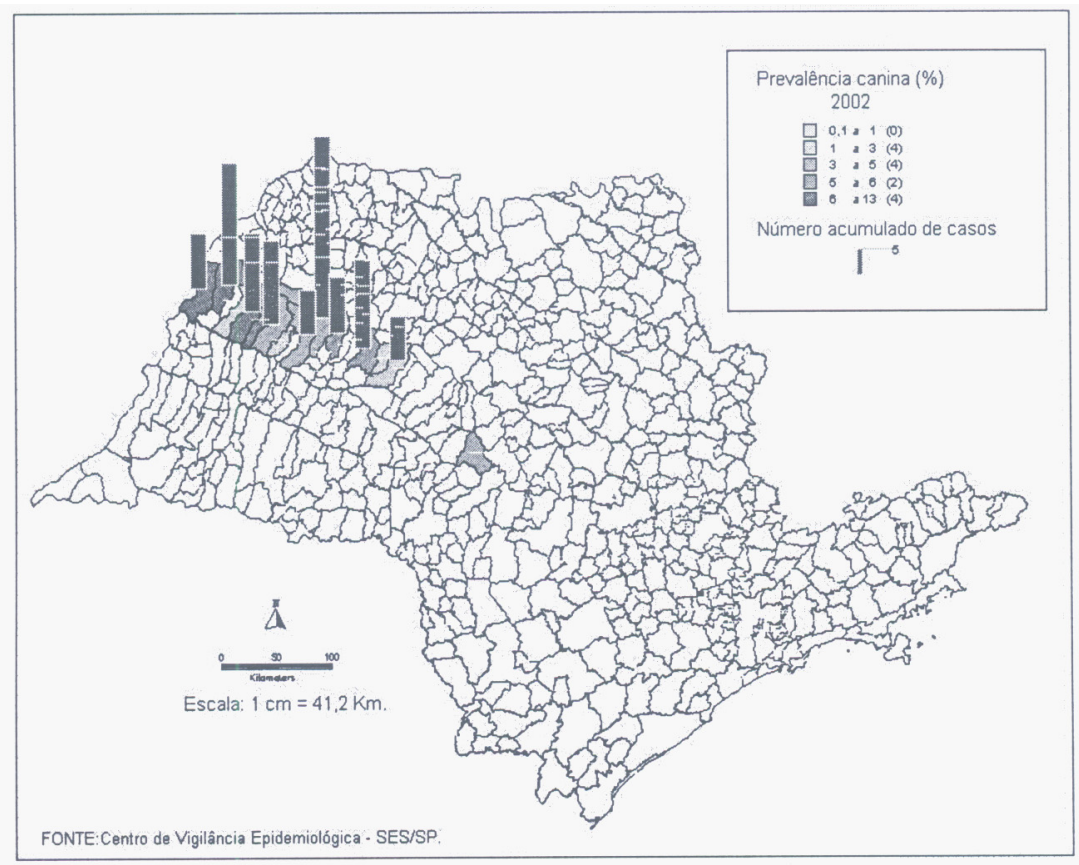

Figura 2 - Distribuição do número acumulado de casos humanos de Leishmaniose Visceral Americana, no período de 1999 a 2002, e prevalência canina, em 2002. Estado de São Paulo.

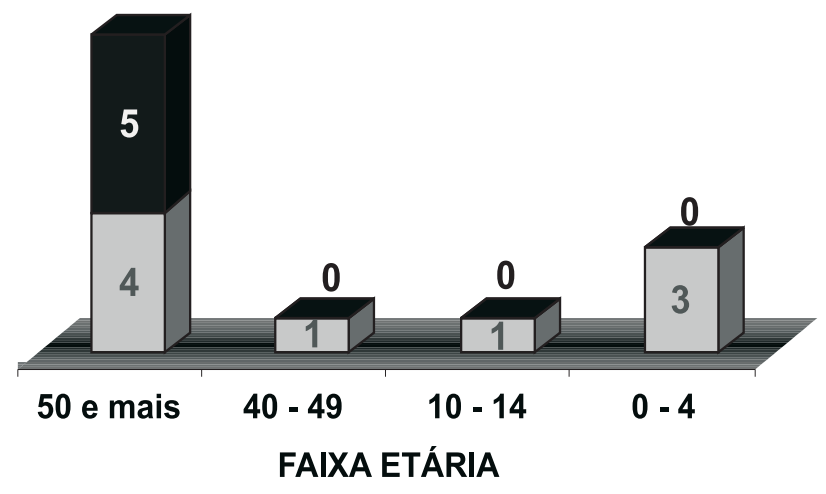

$\square$ LVA $\square$ co-morbidade associada

Figura 3 - Distribuição do Número de Óbitos por Leishmaniose Visceral Americana e Outras Causas Associadas, por faixa etária. Estado de São Paulo, 2001 - 2002.

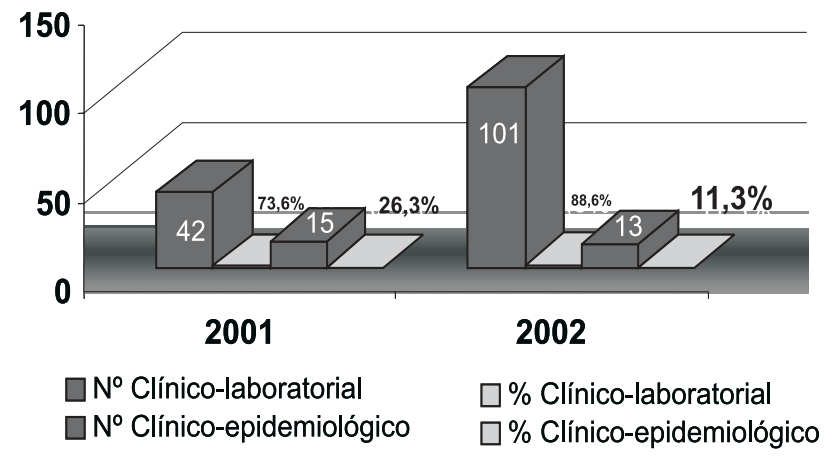

Figura 4 - Distribuição do Número e Percentual de Casos de Leishmaniose Visceral Americana Confirmados segundo Critério Diagnóstico. Estado de São Paulo, 2001 - 2002. de transmissão. Verificou-se, também, a necessidade da implantação de serviço de verificação de óbitos e da realização de treinamentos e de fóruns de discussão com a classe médica a fim de atingir o principal objetivo do programa de controle da LVA no Estado que é a redução da mortalidade pela doença.

\section{REFERÊNCIAS BIBLIOGRÁFICAS}

Camargo-Neves V L F de, Katz G. Leishmaniose visceral americana no Estado de São Paulo. Revista da Sociedade Brasileira de Medicina Tropical; 32 (Supl.II): 63-4, 1999

Camargo-Neves V L F de, Katz G, Rodas L A C, Poletto D W, Lages L C, Spinola R M F, Cruz $0 \mathrm{G}$. Use of spacial analysis tools in the epidemiological surveillance of American visceral leishmaniasis, Araçatuba, São Paulo, Brazil, 1998 - 1999. Cadernos de Saúde Pública, Rio de Janeiro; 17 (5): 1263-7, 2001.

Camargo-Neves V L F de, Rodas L A C, Poletto D W, Gomes A de C. Feeding habit of Lutzomyia longipalpis in Araçatuba county, State São Paulo, Brasil. Entomología y Vectores; 9, (Supl. 1): 63, 2002.

Costa A I P da, Casanova C, Rodas L A C, Galati E A B. Atualização da distribuição geográfica e o primeiro encontro de Lutzomyia longipalpis em área urbana no Estado de São Paulo, Brasil; notas e informações. Revista de Saúde Pública; 31:632-3, 1997

SES -SP Secretaria de Estado de Saúde de São Paulo. II Informe técnico: Leishmaniose Visceral Americana, 2003. Disponível em URL http: \www.sucen.sp.gov.br.

Sherlock IA. Interação Ecológica da Lutzomyia longipalpis com a Leishmania (L.) chagasi na epidemiologia da leishmaniose visceral americana. Revista da Sociedade Brasileira de Medicina Tropical (Supl IV): 579 - 82, 1994.

Tolezano JE, Luvizotto MCR, Uliana SRB, Araújo MFL, Taniguchi HH, Barbosa JAR, Barbosa JER, Pinto PLS, Floeter-Winter L, Shaw JJ. Leishmaniose visceral americana (LVA) em Araçatuba, região Oeste do Estado de São Paulo. Investigações laboratoriais e diagnóstico de uma doença emergente em terras paulistas. Revista da Sociedade Brasileira de Medicina Tropical 32 (Supl): 218, 1999. 